\title{
ANÁLISIS DE ÁCIDOS GRASOS Y ESTEROLES DEL LÍQUEN EVERNIOPSIS TRULLA
}
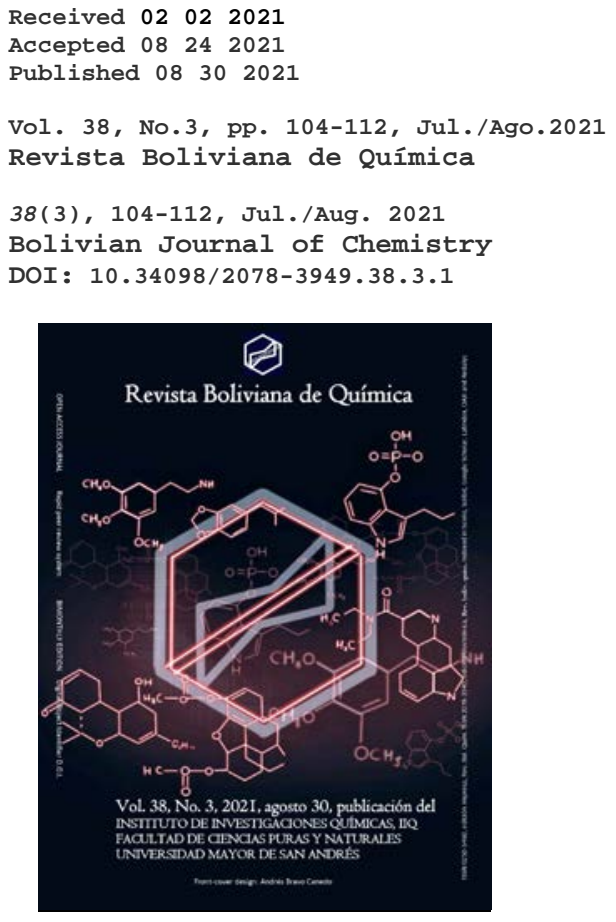

Full original article

Peer-reviewed

\section{Olivio N. Castro Mandujano리 J Jenny L. Álvarez Bautista²}

1. Departamento de Química Orgánica, Facultad de Química e Ingeniería Química, UNMSM. Av. Germán Amezaga 375, Cercado de Lima, Lima, Perú. ocastrom@unmsm.edu.pe

2. Departamento de Química Analítica, Facultad de Química e Ingeniería Química, UNMSM. Av. Germán Amezaga 375, Cercado de Lima, Lima, Perú. jenny.alvarez73@yahoo.com

Keywords: Fatty acids, Sterols, Everniopsis trulla, Gas chromatographic.

Palabras clave: Ácidos grasos, Esteroles, Everniopsis trulla, Cromatografía de gases.

\section{ABSTRACT}

In the present work a chromatographic analysis of lichen Everniopsis trulla was carried out, which was collected in the department of Ancash, province of Asunción. A gaseous chromatographic analysis was carried out, which allowed us to identify the fatty acids and sterols, for which an organic extract was made, applying a classical method. A rapid method was applied to identify other compounds. These two methods, 19 fatty acids, 4 sterols, 15 lipid compounds (esters, alcohols, alkanes, etc.) were identified.

\section{*Correspondent author: ocastrom@unmsm.edu.pe}

\section{RESUMEN}

En el presente trabajo se realizó un análisis cromatográfico del liquen Everniopsis trulla, que fue recolectado en el departamento de Ancash, provincia de Asunción; por un lado, se realizó un análisis cromatográfico gaseoso el cual nos permitió identificar los ácidos grasos y esteroles, para ello, se realizó un extracto orgánico, aplicando un método

Downloadable from: Revista Boliviana de Química http://www.bolivianchemistryjournal.org
104 Volumen 38 N³. Año 2021 http://www.scribd.com/bolivianjournalofchemistry 

identificó a 19 ácidos grasos, 4 esteroles, y 15 compuestos lipídicos (esteres, alcoholes, alcanos, etc.).

\section{INTRODUCCIÓN}

Los lípidos se definen como sustancias derivadas de tejidos vivos que pueden extraerse o solubilizarse en disolventes orgánicos de baja polaridad. Esta definición bastante flexible abarca una amplia gama de compuestos que van desde moléculas de bajo peso molecular como los ácidos grasos, esteroles hasta complejos como los glicolípidos de alto peso molecular [1].

Por otro lado, Hostettmann 2008 [2], comenta que el progreso de la fitoquímica de los productos naturales esta siempre ligado a las innovaciones de la tecnología analítica [2]. La identificación de metabolitos lipídicos de extractos orgánicos de los líquenes junto con las técnicas sofisticadas como las que se disponen actualmente en los laboratorios, como la cromatografía de gases acoplada a la espectrometría de masas (GC-MS). Con la cual, ahora es posible producir directamente una fase gaseosa de iones moleculares para la mayoría de los lípidos, tanto si son ácidos grasos simples o sustancias lipídicas más complejas, todo esto da como resultado una información estructural más completa sobre los constituyentes lipídicos del liquen [2, 3].

Según la búsqueda bibliográfica realizada, no hay estudios lipídicos del liquen Everniopsis trulla, pero cabe resaltar la investigación de Ramaut [4], 1978, realizó solo cromatografía de capa delgada CCD, del extracto etanólico. Elix [5], 1993, hace una revisión de la familia Parmeliaceae e investigó a la E. trulla pero no menciona ningún componente lipídico, la publicación de Castro [6] en 2017, hizo un análisis metabolómica del liquen E. trulla, empleando el UHPLC-DAD-MS, el cual le permitió identificar la presencia de dos compuestos aromáticos simples, seis derivados lipídicos, ocho depsidonas, trece dépsidos, un cromona, dos difeniléteres y un dibenzofurano identificado. En este sentido, nuestro objetivo de la presente investigación es identificar los componentes lipídicos por análisis CG-MS.

\section{EXPERIMENTAL}

\section{Material Biológico}

El liquen en estudio ha sido recolectado en el departamento de Ancash, provincia de Asunción, distrito de Chacas, pueblo de Canchas; a una altitud $3427 \mathrm{msnm}$. La fecha de la primera recolección fue el día 5 de mayo de 2015; las coordenadas del lugar de recolección son: $9^{\circ} 09^{\prime} 56.59^{\prime \prime} \mathrm{S}$; 77²2’08.40" O. El nombre científico, fue identificado por la Dra. Magda Chanco (Museo de Historia Natural de la UNMSM).

\section{Marcha Fitoquímica y Reacciones de Coloración}

Para un análisis preliminar se pueden realizar diferentes ensayos directos a los extractos disponibles, esto es bueno usarlo cuando nos interesa conocer los tipos de compuestos que contiene el liquen, así de esta forma dirigir mejor los objetivos y/o definir las estrategias a realizar y aislar los compuestos. Por ello, se realizó la marcha fitoquímica como indica la referencia [7]. También se realizó diferentes reacciones de coloración específicos para compuestos liquénicos [7].

\section{Análisis de Ácidos Grasos por CG}

Se aplicó el método clásico, el cual consta de varias etapas (aproximadamente 8 etapas). Se pesó $200 \mathrm{mg}$ de la muestra (extracto con $\mathrm{CHCl}_{3}-\mathrm{MeOH}$ 1:1), luego, se saponificó con $\mathrm{KOH} \mathrm{0,5} \mathrm{N}$, en baño de agua, a $55^{\circ}$ C, por 20 min; la saponificación liberó los ácidos grasos de la muestra, las que se neutralizó por adición de $5 \mathrm{~mL}$ de $\mathrm{HCl}$ diluido (1:1), los ácidos se extraen con $10 \mathrm{~mL}$ de éter de petróleo. Este primer extracto, se lavó por agitación con $10 \mathrm{~mL}$ de agua, el extracto etéreo se seca con sulfato de sodio anhidro, se evaporó a sequedad con corriente de $\mathrm{N}_{2}$. El residuo se disolvió en $10 \mathrm{~mL}$ de éter de petróleo; se tomó una alícuota de $1 \mathrm{~mL}$, la que se evaporó a sequedad en presencia de nitrógeno, se agregó $10 \mathrm{~mL}$ de $\mathrm{HClO}_{4}$ al 5\% en metanol y se calentó a $55^{\circ} \mathrm{C}$ por 5 min. Los ésteres se extrajeron con éter, se lavaron y secaron como en el primer

Downloadable from: Revista Boliviana de Química http://www.bolivianchemistryjournal.org
105

Volumen 38 N³. Año 2021

http://www.scribd.com/bolivianjournalofchemistry 
extracto, quedando listos para el análisis en el CG [8]. Luego, se tomó una alícuota de $1 \mu \mathrm{L}$, se inyectó en el cromatógrafo, de igual forma en paralelo se inyectaron los estándares, bajo las siguientes condiciones: Equipo: cromatógrafo Hewlett Packard-HP-5890, serie II; detector FID; columna HP - 5 (5\%Fenil metil

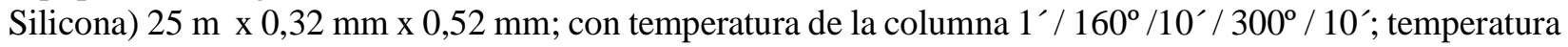
del inyector $280^{\circ} \mathrm{C}$; temperatura del detector $300^{\circ} \mathrm{C}$; gas carrier He; Flujo del $\mathrm{H}_{2}$ a $1 \mathrm{~mL} / \mathrm{min}$; volumen inyectado es $1 \mu \mathrm{L}$ y Split: 1:100.

\section{Análisis de Esteroles por CG}

El método para el análisis de esteroles se inició saponificando $100 \mathrm{mg}$ del extracto $\mathrm{CHCl}_{3}$-Etanol, con $2 \mathrm{~mL}$ de $\mathrm{KOH} \mathrm{50 \%} \mathrm{y} 8 \mathrm{~mL}$ de etanol 95\%, se agitó 10 minutos con magneto en plancha sin calentamiento. Se llevó a un baño maría a $90^{\circ} \mathrm{C}$ durante 1 hora, se agita 10 minutos con magneto en plancha sin calentamiento, luego, se enfrió. Luego, se agregó $5 \mathrm{~mL}$ de agua destilada, transfirió a una pera de decantación de $100 \mathrm{~mL}$, se extrajo con $5 \mathrm{~mL}$ de hexano 4 veces. Se agregó un poco de $\mathrm{Na}_{2} \mathrm{SO}_{4}$ para secar el extracto hexánico, se filtró y el filtrado se secó bajo atmósfera de nitrógeno. A esta muestra se agregó $1000 \mu \mathrm{L}$ de hexano, de esta solución se inyectó $1 \mu \mathrm{L}$ al cromatógrafo de gases [8].

\section{Análisis de componentes lipídicos por un método directo}

El método aplicado es un método rápido, donde se trabaja con un extracto de diclorometano. Se pesó 5 gramos, se añadió $15 \mathrm{~mL}$ de $\mathrm{CH}_{2} \mathrm{Cl}_{2}$, se colocó en el ultrasonido por 10 minutos a temperatura ambiente, luego, este extracto lipídico se concentró hasta sequedad, luego al residuo se añadió hexano $5 \mathrm{ml}$ y se llevó al ultrasonido por 2 minutos; a esta solución hexánica, se analizó en el equipo, se inyectó al cromatógrafo (Agilent GC-MS 5973), pero, ahora este equipo este acoplado a un espectrómetro de masas y tiene una base de datos NIST. [9]. Las condiciones cromatográficas son las siguientes: tipo de columna: capilar; clase de columna: estándar no polar; gas carrier: $\mathrm{H}_{2}$; fase activa: $\mathrm{DB}-1$; longitud de la columna: $30 \mathrm{~m}$; diámetro de la columna: $0,2 \mathrm{~mm}$; temperatura de inicio: $50^{\circ} \mathrm{C}$; temperatura final: $300^{\circ} \mathrm{C}$ y velocidad de calentamiento: $5 \mathrm{~K} / \mathrm{min}[10-12]$.

\section{RESULTADOS Y DISCUSIÓN}

\section{Resultados de la marcha fitoquímica y reacciones de coloración del liquen}

La marcha fitoquímica realizada en forma general según referencia (con la única modificación de que se empleó 100 gramos de muestra seca y molida, en vez de 50 gramos), dio como resultado que el liquen, la Everniopsis trulla, contiene compuestos liquénicos ácidos fenólicos, dépsidos, depsidonas, ácidos úsnico y además contiene otros metabolitos secundarios como esteroides, triterpenos, y otros que se indican en la tabla 1 , y no contiene alcaloides.

Tabla 1. Resultados de la marcha fitoquímica y de las reacciones de coloración.

\begin{tabular}{|c|c|c|c|}
\hline COMPUESTOS & REACTIVOS & COMPUESTO & RESULTADO \\
\hline $\begin{array}{l}\text { Metabolitos } \\
\text { secundarios }\end{array}$ & $\begin{array}{l}\mathrm{FeCl}_{3} \\
\text { Ninhidrina } \\
\text { Shinoda } \\
\text { Borntrager } \\
\text { Dragendorff } \\
\text { Mayer } \\
\text { Liebermann-Burchard }\end{array}$ & $\begin{array}{l}\text { Fenoles y taninos } \\
\text { Aminoácidos } \\
\text { Flavonoides } \\
\text { Antraquinonas } \\
\text { Alcaloides } \\
\text { Alcaloides } \\
\text { Esteroides y triterpenos }\end{array}$ & $\begin{array}{c}+++ \\
+ \\
- \\
- \\
- \\
- \\
++\end{array}$ \\
\hline $\begin{array}{l}\text { Compuestos } \\
\text { liquénicos }\end{array}$ & $\begin{array}{l}\mathrm{FeCl}_{3} \\
\mathrm{KOH} \\
\mathrm{Ca}(\mathrm{ClO})_{2} \\
\mathrm{KOH}+\mathrm{Ca}(\mathrm{ClO})_{2}\end{array}$ & $\begin{array}{l}\text { Ácidos fenólicos } \\
\text { Depsidonas } \\
\text { Dépsidos } \\
\text { Ácido úsnico }\end{array}$ & $\begin{array}{c}+ \\
+ \\
++ \\
+++\end{array}$ \\
\hline
\end{tabular}

Downloadable from: Revista Boliviana de Química http://www.bolivianchemistryjournal.org
106

Volumen 38 N³. Año 2021

http://www.scribd.com/bolivianjournalofchemistry 
Estos resultados, en parte concuerda con las dos únicas investigaciones de la misma especie de Ramaut (1978) [4] y Elix (1993) [5], en donde por un lado Ramaut (1978), realizó sólo CCD del extracto etanólico del líquen Everniopsis trulla (Apurímac-Perú), y determinaron que la Everniopsis trulla contiene ácido úsnico, atranorina, cloroatranorina y dos componentes no identificados. Además, Elix (1993) [5] hizo un estudio botánico y menciona que la composición química de la Everniopsis trulla es: atranorina y ácido úsnico en la parte superficial del talo; en las paredes celulares hay polisacáridos no determinados; además, contiene triterpenos y $\beta$-orcinol depsidonas, en la parte medular del liquen.

\section{Resultados del análisis de ácidos grasos por CG (Método Clásico)}

Este es un método usado frecuentemente, pero tiene la desventaja que se emplean patrones cuando no se tiene el equipo acoplado al espectrómetro de masas, son muchas etapas (alrededor de 8), genera bastante residuos orgánicos, etc. para este método empleamos el equipo HP 5890 y tuvimos que pasar también los estándares a las mismas condiciones de trabajo.

El procedimiento experimental nos garantiza tener a los ácidos grasos libres por saponificación (cuando se añadió el $\mathrm{KOH}$ ), previamente fue derivatizado para obtener un extracto bruto de ácidos grasos; luego, se inyecta al equipo y así se logra identificar a los ácidos grasos comparándolos con los patrones que se tiene a disposición. En la figura 1, se observa el cromatograma de los estándares y en la figura 2, se observa el cromatograma del liquen en estudio, en donde se identifica a los ácidos grasos presente en la planta liquénica según su tiempo de retención, esta información se resume en la tabla 2; los valores y el nombre del ácido graso concuerda con la referencia bibliográfica [8, 9]. Con ayuda de estándares se detectaron 17 ácidos grasos (figura 2), de los cuales en mayor proporción se encuentran el ácido palmítico, ácido cis-oleico y ácido linoleico. De los resultados obtenidos por CG vemos que la E. trulla contiene los ácidos grasos saturados como el palmítico (16:0) y esteárico (18:0), además, contiene los ácidos grasos insaturados linoleico (18:2) y cis-oleico (18:1) ver tabla 2.

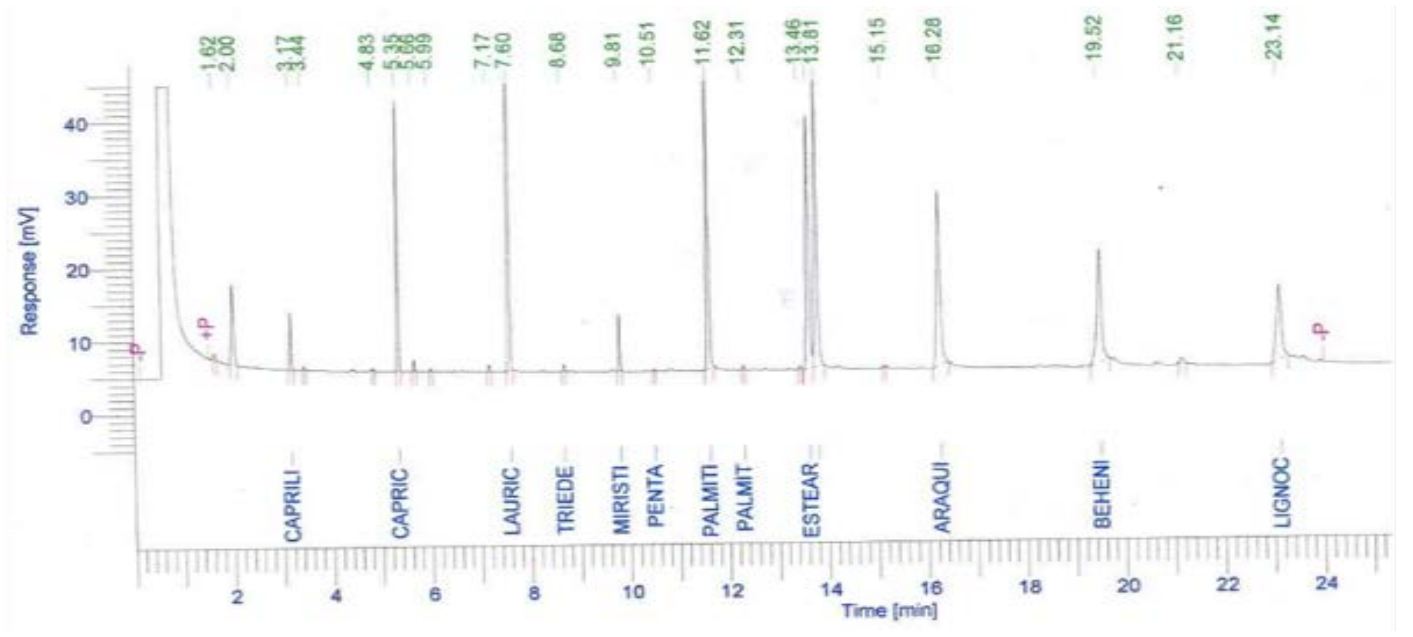

Figura 1. Cromatograma de los estándares de ácidos grasos

En la presente investigación, también, se encontraron ácidos grasos minoritarios como el acido caprílico (8:0), cáprico (10:0), laúrico (12:0), tridecanoico (C13:0), mirístico (14:0), pentadecanoico (15:0), palmitoleico (16:1), heptadecanoico (C17:0), linolénico (18:3), nonadecaonato, araquídico (20:0), eicosenoato, heneicosanoico (C21:0), behénico (22:0) y lignocérico, todos estos componentes van de acuerdo a los reportados en la bibliografía [9].

\section{Resultados del análisis de esteroles por CG}

El procedimiento experimental nos garantiza inyectar un extracto orgánico bruto en esteroles casi en su totalidad. Así cuando analizamos los cromatogramas, ver figura 3 y 4, tenemos al cromatograma de los cuatro estándares (brassicasterol, campesterol, estigmasterol y $\beta$-sitosterol) y de la muestra respectivamente, corridos a las mismas condiciones. En la tabla 3, se muestran las cantidades relativas encontradas de esteroles analizados; además, en la

Downloadable from: Revista Boliviana de Química http://www.bolivianchemistryjournal.org
107

Volumen 38 Nº3. Año 2021

http://www.scribd.com/bolivianjournalofchemistry 
REVISTA BOLIVIANA DE QUÍMICA

ISSN 0250-5460 Rev. Bol. Quim. Paper edition

ISSN 2078-3949 Rev boliv, quim. Electronic edition

olivio N. Castro Mandujano et Jenny L. Álvarez Bautista RBQ Vol.38, No.3, pp. 104-112, 2021

figura 4, se observa que aparte de estos esteroles estándares existen otras señales donde falta identificar a estos esteroles, pero que con una análisis cromatográfico CG-SM, si se podría identificar y cuantificar a todos los esteroles contenidos en el liquen (ver tabla 3), estos componentes y su valores concuerda con la bibliografía [10,11].

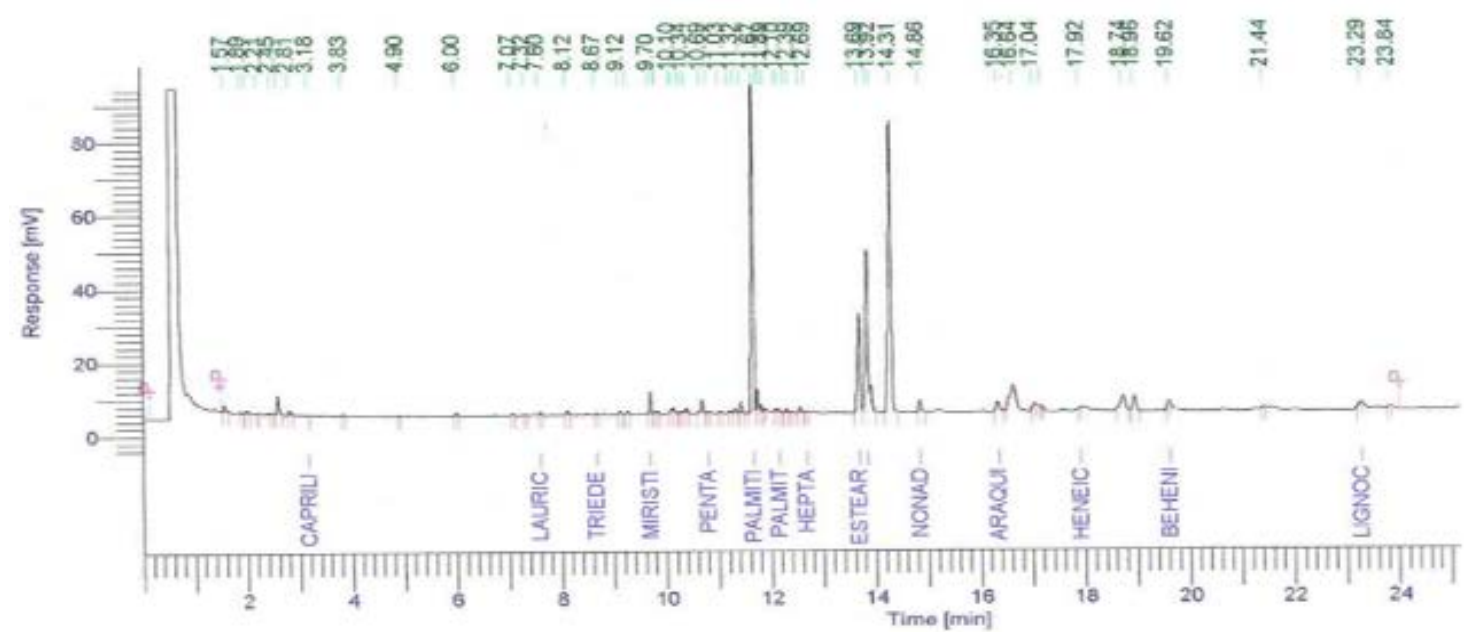

Figura 2. Cromatograma de los ácidos grasos del liquen Everniopsis trulla

Tabla 2. Contenido de ácidos grasos en el liquen Everniopsis trulla

\begin{tabular}{ccccc}
\hline $\begin{array}{c}\text { Nombre del ácido } \\
\text { graso }\end{array}$ & $\begin{array}{c}\text { Cantidad } \\
\text { relativa en el liquen \% }\end{array}$ & & $\begin{array}{c}\text { Nombre del ácido } \\
\text { graso }\end{array}$ & $\begin{array}{c}\text { Cantidad } \\
\text { en el liquen \% }\end{array}$ \\
\cline { 1 - 2 } Ác. lignocérico & 0,14 & & Ac. esteárico & 8,71 \\
Ác. behémico & 0,21 & & Ác.heptadecanoico & 0,42 \\
Ác.heneicosanoico & 0,04 & Ác. palmitoleico & 0,20 \\
Ác. araquidico & 0,08 & & Ác. palmitico & 26,68 \\
Ác. linolénico & 1,19 & Ác.pentadecanoico & 0,65 \\
Ác. linoleico & 23,32 & & Ác. miristico & 0,70 \\
Ac. cis-oleico & 15,31 & & Ác. tridecanoico & 0,02 \\
Ac. esteárico & 8,71 & & Ác. laúrico & 0,11 \\
Ác.heptadecanoico & 0,42 & & Ác. cáprico & 0,95 \\
& & & Ác. caprílico & 0,05 \\
\hline
\end{tabular}

Tabla 3. Cantidad relativa de esteroles que contiene el liquen

\begin{tabular}{lcc}
\hline Esteroles & $\begin{array}{c}\text { Tiempo de retención en } \\
\text { minutos }\end{array}$ & \% \\
\hline Brassicasterol & 21.501 & 5.91 \\
Campesterol & 22.361 & 3.31 \\
Estigmasterol & 22.892 & 4.91 \\
$\beta$-sitosterol & 23.826 & 4.94 \\
\hline
\end{tabular}

Downloadable from: Revista Boliviana de Química http://www.bolivianchemistryjournal.org
108

Volumen 38 Nº3. Año 2021

http://www.scribd.com/bolivianjournalofchemistry 


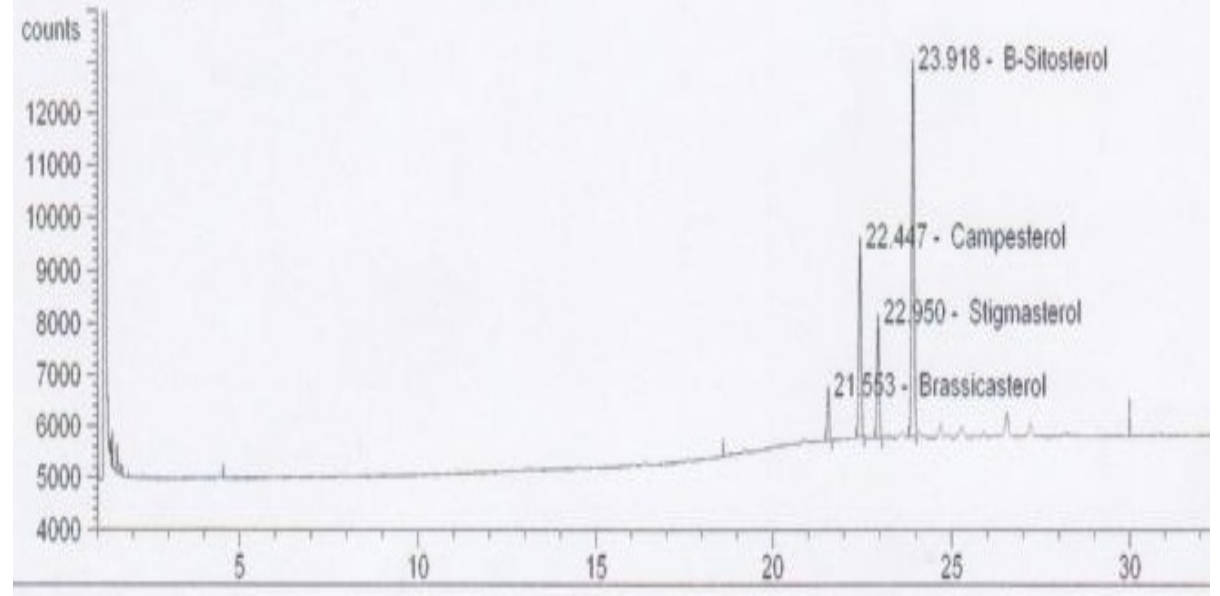

Figura 3. Cromatograma de CG de esteroles estándares

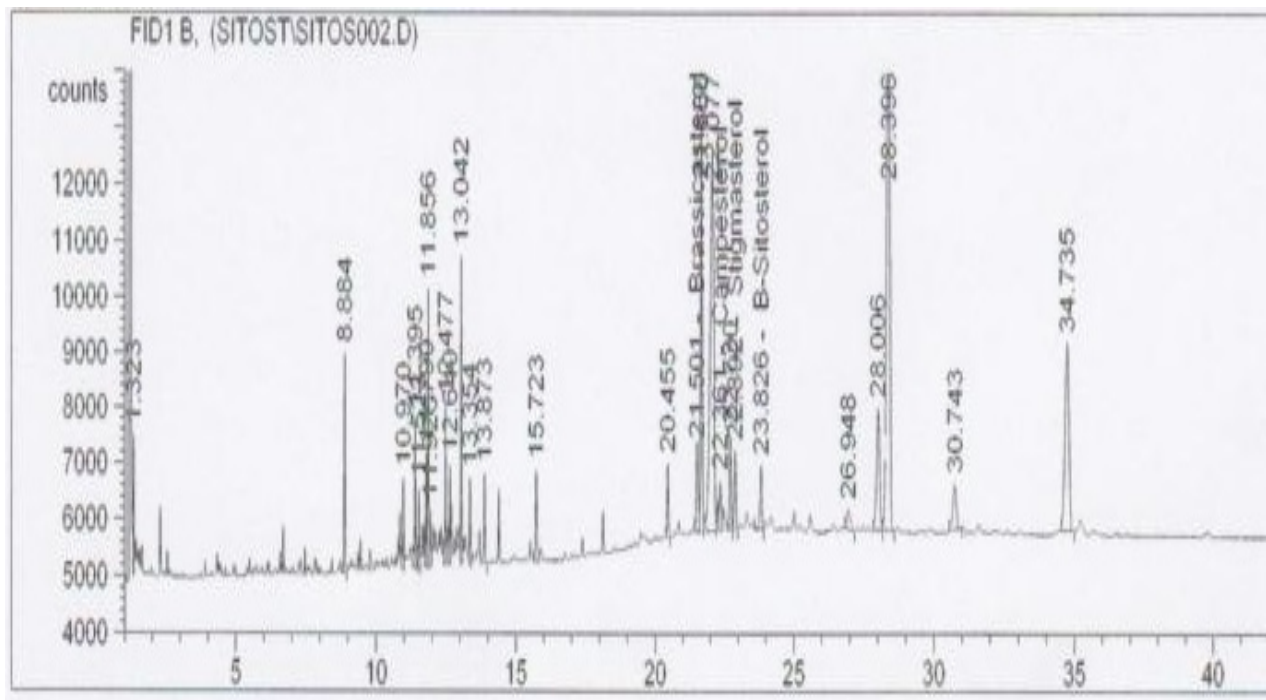

Figura 4. Cromatograma de esteroles de CG de la Everniopsis trulla

\section{Resultados del análisis lipídico del liquen mediante el método directo}

El método aplicado es un método rápido en el la cual se realizó un extracto diclorometano y luego de concentrar otro extracto con hexano, finalmente, de este último extracto se inyecta el equipo; todo aquello permitió analizar los componentes lípidos, componentes apolares $(13$-metil, $(8 \beta, 13 \beta)$ 17-norkau-15-eno) y sustancias de baja polaridad como el acido metil ester, (z)-9-hexadecenoico. En la figura 5, se observa el perfil lípidos y allí hay varias señales que todavía falta identificar. Si bien es un método sencillo, pero no es especifico, es decir, es una extracción de todos los tipos de componentes de baja polaridad y/o que son solubles en diclorometano [10-12].

El cromatograma de gases del extracto de diclorometano de la E. trulla se presenta en la Figura 5. A manera de ejemplo de los compuestos obtenidos, sus estructuras químicas de algunos de ellos se muestran en la figura 6 y 7 , en donde se identifican solo a 15 picos, los más altos, pero como se observa el cromatograma hay otros picos de baja intensidad y que estos faltan identificar. Recordemos que al hacer el procedimiento clásico de análisis para ácidos grasos y esteroles es posible que se haya formado algunos derivados propios de la reacción de derivatización. En cambio, en este análisis con hexano o diclorometano solo se hizo una extracción orgánica y a temperatura ambiente, entonces en este caso no se ha formado ningún derivado. En la figura 6, se encuentran los espectros de masas y sus

Downloadable from: Revista Boliviana de Química http://www.bolivianchemistryjournal.org
109

Volumen 38 №3. Año 2021

http://www.scribd.com/bolivianjournalofchemistry 
REVISTA BOLIVIANA DE QUÍMICA

ISSN 0250-5460 Rev. Bol. Quim. Paper edition

ISSN 2078-3949 Rev. boliv. quim. Electronic edition

olivio N. Castro Mandujano et Jenny L. Álvarez Bautista RBQ Vol.38, No.3, pp. 104-112, 2021

estructuras químicas de algunas sustancias de los 15 compuestos lipídicos identificados. Por otro lado, en la tabla 4 se muestran los compuestos lipídicos identificados con algunas características [10-12].

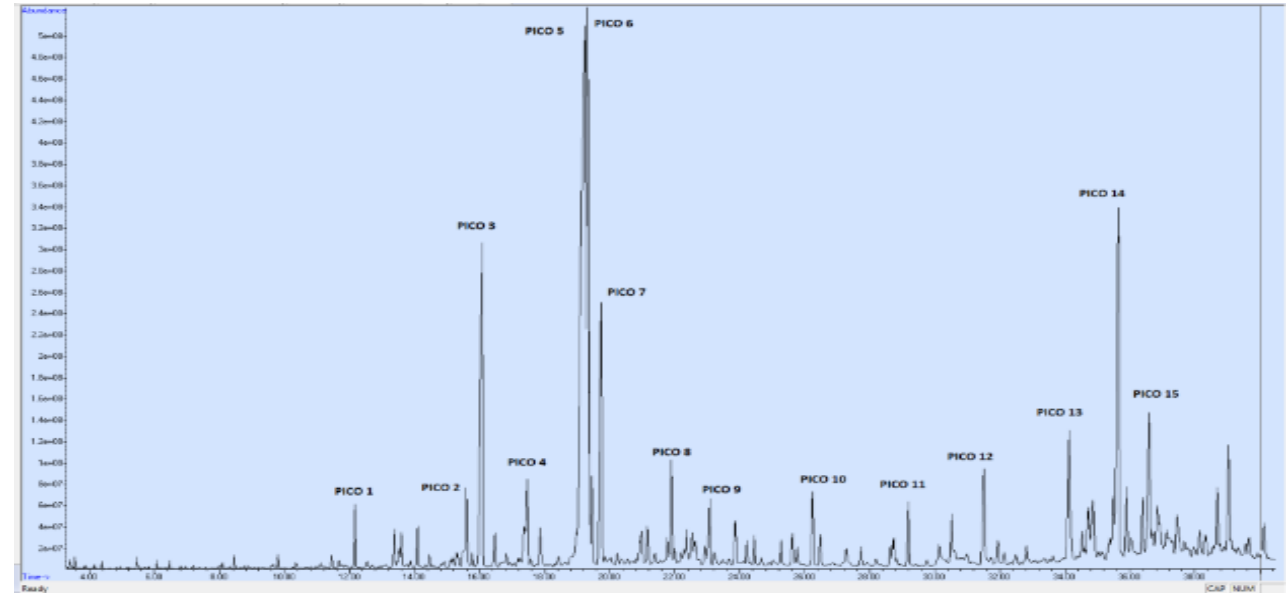

Figura 5. Cromatograma del análisis lipídico del liquen E. trulla

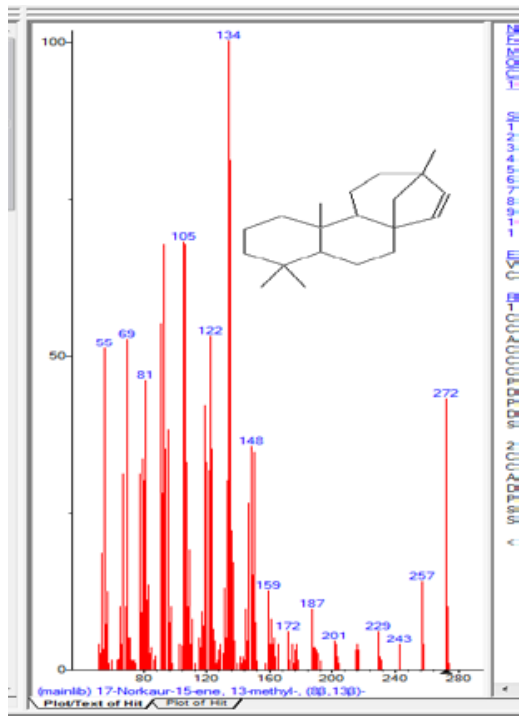

pico 4: 13-metil, $(8 \beta, 13 \beta)$ 17-norkau-15-eno

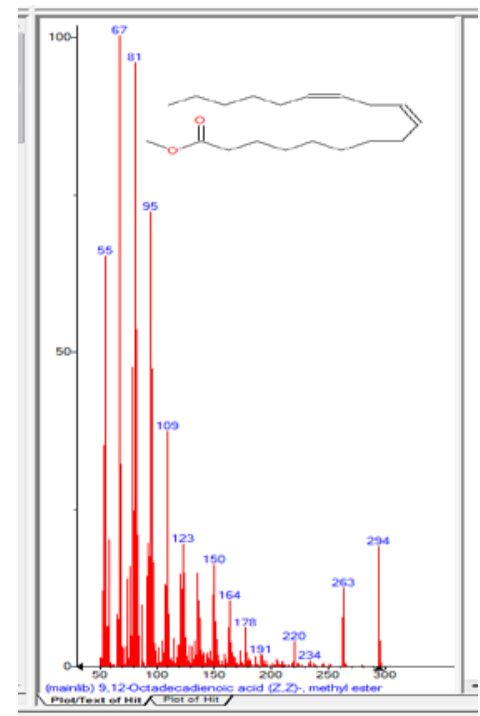

pico 5: acido metil ester $(z, z)-9,12-$ octadecadenoico

Figura 6. Espectros de masas de los picos 1-6 del liquen E. trulla

Finalmente cabe mencionar que debido a sus componentes identificados de ácidos grasos y esteroles esta especie liquénica aporta una composición para la dieta de los humanos, esta sería la razón porque muchos de estos líquenes son consumidos en Japón y China [12].

\section{CONCLUSIÓN}

Se realizó el análisis lipídico con dos diferentes métodos y se logró identificar a los componentes lipídicos del liquen E. trulla, estos son: 19 ácidos grasos, 4 esteroles, 15 compuestos lipídicos (esteres, alcoholes, alcanos, etc.).

Downloadable from: Revista Boliviana de Química http://www.bolivianchemistryjournal.org
110

Volumen 38 №3. Año 2021

http://www.scribd.com/bolivianjournalofchemistry 
ISSN 0250-5460 Rev. Bol. Quim. Paper edition

ISSN 2078-3949 Rev, boliv. quim. Electronic edition

pico 14: $17 \alpha, 21 \beta, 2 \beta, 30$-bisnorhopano

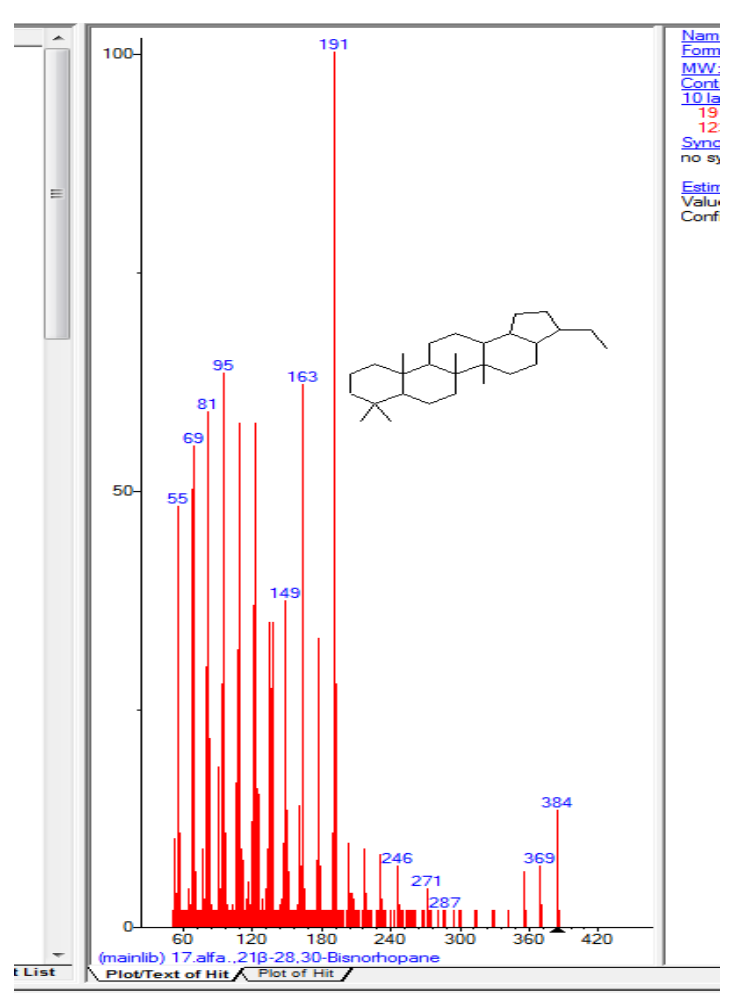

Figura 7. Espectros de masas de los picos 13-15 del liquen E. trulla

Tabla 4. Componentes lipídicos del liquen Everniopsis trulla

\begin{tabular}{|c|c|c|c|c|}
\hline PICO & $\begin{array}{l}\text { Tiempo de } \\
\text { retención } \\
\text { tr en min }\end{array}$ & COMPUESTO & FM & PM \\
\hline 1 & 12,143 & metil tetradecanoato & $\mathrm{C}_{15} \mathrm{H}_{30} \mathrm{O}_{2}$ & 342,22 \\
\hline 2 & 15,818 & $\begin{array}{l}\text { acido metil ester ( } \mathrm{z})-9 \text { - } \\
\text { hexadecenoico }\end{array}$ & $\mathrm{C}_{17} \mathrm{H}_{32} \mathrm{O}_{2}$ & 268,24 \\
\hline 3 & 16,082 & $\begin{array}{l}\text { acido metil ester, (z)-9- } \\
\text { hexadecenoico }\end{array}$ & $\mathrm{C}_{17} \mathrm{H}_{32} \mathrm{O}_{2}$ & 268,24 \\
\hline 4 & 17,225 & acido metil ester hexadecanoico & $\mathrm{C}_{17} \mathrm{H}_{34} \mathrm{O}_{2}$ & 270,26 \\
\hline 5 & 19,117 & 13-metil, $(8 \beta, 13 \beta)$ 17-norkau-15-eno & $\mathrm{C}_{20} \mathrm{H}_{32}$ & 272,25 \\
\hline 6 & 19,206 & $\begin{array}{l}\text { acido metil ester (z, z)-9,12- } \\
\text { octadecanoico }\end{array}$ & $\mathrm{C}_{19} \mathrm{H}_{34} \mathrm{O}_{2}$ & 294,26 \\
\hline 7 & 20,188 & $\begin{array}{l}\text { acido metil ester (z)-9- } \\
\text { octadecenoico }\end{array}$ & $\mathrm{C}_{19} \mathrm{H}_{36} \mathrm{O}_{2}$ & 296,27 \\
\hline 8 & 21,872 & metil estearato & $\mathrm{C}_{19} \mathrm{H}_{38} \mathrm{O}_{2}$ & 298,29 \\
\hline 9 & 22,852 & acido metil ester araquidonico & $\mathrm{C}_{21} \mathrm{H}_{34} \mathrm{O}_{2}$ & 318,25 \\
\hline
\end{tabular}

Downloadable from: Revista Boliviana de Química http://www.bolivianchemistryjournal.org
111

Volumen 38 N³. Año 2021

http://www.scribd.com/bolivianjournalofchemistry 


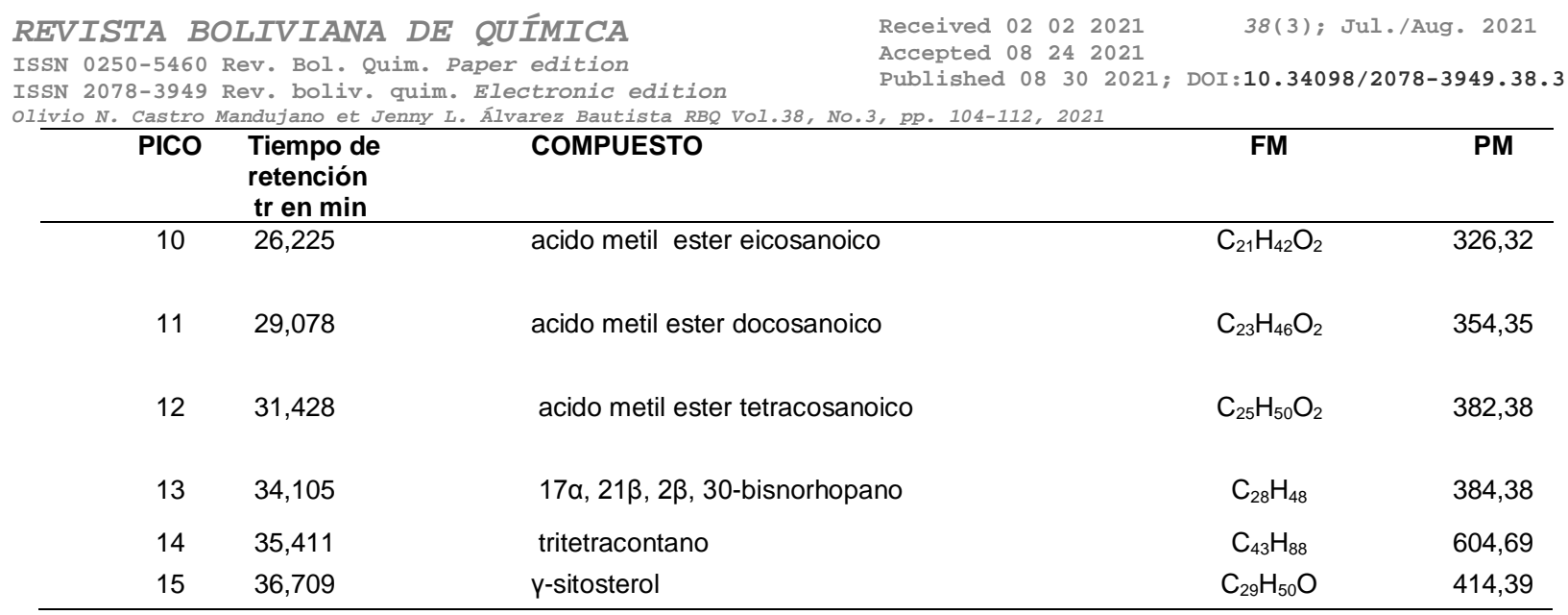

\section{RECONOCIMIENTOS}

Se agradece a la Dra. Magda Chanco, por la identificación botánica; al Quím. César Poma Pando, Jefe del Laboratorio de Análisis Servicios Analíticos Generales SAC, Lima-Perú.

\section{REFERENCIAS}

1. Murphy, R., Fiedler, J., Hevko, J. 2001, Analysis of Nonvolatile Lipids by Mass Spectrometry, Chem. Rev., 101(2), 479-526. DOI: https://doi.org/10.1021/cr9900883

2. Hosterttmann, K., Gupta, M., Marston, A., Ferreira, E. 2008, Handbook of strategies for the isolation of bioactive natural products, SECAD y CYTED, Serie Ciencia y Tecnología No. 160, Bogotá.

3. Rankovic, B. Lichen secondary metabolites, Springer, Cham, 2014, Germany. DOI: 10.1007/978-3-319-13374-4

4. Ramaut J L, Brouers M, Serusiaux E, Corvisier M. 1978, Separation of mixtures of atranorina and chloroatranorin by thin layer chromatograph, Journal Chromatography, 155, 450-453.

5. Elix, J. 1993, Progress in the generic delimitation of Parmelia sensu lato lichens (Ascomycotina: Parmeliaceae) and a Synoptic key to the Parmeliaceae, The Briologist, 96(3), 359-383. DOI: https://doi.org/10.2307/3243867

6. Castro, O., Benites, B., Rodilla, J., 2017, Metabolomic Analysis of the Lichen Everniopsis trulla using Ultra High Performance Liquid Chromatography-Q uadrupole- 0 rbitrap M ass Spectrom etry (U HPLC-Q-0 T-M S), Chromatographia, 80, 967-973. DOI: 10.1007/s10337-017-3304-4

7. Castro, O. Investigación fitoquímica de los líquenes. editorial académica española, 2016, Riga-Letonia, Unión Europea.

8. Molina, M. C., Crespo, A., Vicente, C., Elix, J. A. 2003, Differences in the composition of phenolics and fatty acids of cultured mycobiont and thallus of Physconia distorta, Plant Physiology and Biochem, 41(2), 175-180. DOI: https://doi.org/10.1016/S0981-9428(02)000177

9. Sassaki, G., et al. 1999, Glycosyldiacylglycerolipids from the Lichen Dictyonema glabratum. J. Nat. Prod. 62(6), 844-847, DOI: https://doi.org/10.1021/np980547f

10. Safe, St., Safe, L., Maass, W. 1975, Sterols of three lichen species: Lobaria pulmonaria, Lobaria scrobiculata and Usnea longissima, Phytochemistry, 14(8), 1821 - 1823. DOI: https://doi.org/10.1016/0031-9422(75)85302-7

11. Wojciechowski, Z., Goad, J., Goodwin, T. 1973, Sterols of the lichens, Prog. Phytochem., 12(6), 1433 - 1436, DOI: https://doi.org/10.1016/0031-9422(73)80579-5

12. Jiao, J., Zhang, Y. 2013, Transgenic Biosynthesis of Polyunsaturated Fatty Acids: A Sustainable Biochemical Engineering Approach for Making Essential Fatty Acids in Plants and Animals, Chem. Rev., 113(5), 3799-3814. DOI: https://doi.org/10.1021/cr300007p

Downloadable from: Revista Boliviana de Química http://www.bolivianchemistryjournal.org
112

Volumen 38 No3. Año 2021

http://www.scribd.com/bolivianjournalofchemistry 